\title{
ELECTRONIC REPORT OF THE NSW HEALTH SURVEYS 1997 AND 1998
}

\section{Louisa Jorm \\ Epidemiology and Surveillance Branch NSW Department of Health}

The electronic report presents the combined results of health surveys conducted in NSW in 1997 and 1998. It is the most comprehensive such report ever produced in Australia, and consists of more than 6000 web pages.

The surveys were conducted by telephone, using computer-assisted telephone interviewing (CATI). More than 35,000 randomly selected NSW residents aged 16 years and over participated. These people each gave around 25 minutes of their time to help build a detailed picture of the health of the adult population.

New topic areas in this report include:

- oral health

- health-related quality of life measured using the EQ-5D instrument
- self-reported Pap test screening rates

- motorbikes on rural properties

- fences on rural properties.

The report also contains breakdowns for most topic areas by:

- indigenous status

- country of birth

- language spoken at home

- language of interview

- socioeconomic disadvantage

- self-rated health

- geographic remoteness.

Printable versions of each page of the report, and data in spreadsheet form, can be downloaded from internal.health.nsw.gov.au/public-health/nswhs or www.health.nsw.gov.au/public-health/nswhs.

\section{'EVIDENCE FROM SYSTEMATIC REVIEWS OF RESEARCH RELEVANT TO IMPLEMENTING THE WIDER PUBLIC HEALTH AGENDA'}

\section{Peter Sainsbury}

Division of Population Health

Central Sydney Area Health Service

If you are involved in preparing a policy or a plan that concerns public health, in its broadest possible meaning, and you do not consult this report you will now be negligent. Prepared by contributors to the Cochrane and Campbell Collaborations and published by the NHS Centre for Reviews and Dissemination, University of York, UK, in August 2000, this mighty book is an absolutely essential resource for all public health workers and health service planners.

The first four sections of the report present the evidence from systematic reviews of specific interventions concerning cancer, coronary heart disease and stroke, accidents, and mental health. Each section has four subsections covering social and economic, environmental, personal behaviour, and services interventions. So, for instance, concerning providing 'incentives to employees to cycle or walk to work, or leave their cars at home' to reduce heart disease or stroke, the only review found concluded that 'public health exercise promotion strategies aimed at modifying the environment, to encourage walking and cycling, are likely to reach a greater proportion of the inactive population than efforts that aim to increase the use of exercise facilities'. Readers are also informed where there is no evidence from reviews to support an intervention, where no reviews have been performed and where a review is in progress.

The final three sections of the report, organised differently, present the review evidence for interventions involving education, social care and social welfare, and crime, drugs and alcohol.

The authors emphasise that the report does not tell you what to do, but they hope that it will help readers to answer the following questions:

- Which policies might be prioritised because research evidence suggests that they are likely to succeed in achieving specific public health goals?

- Having prioritised policy areas for public health investment, how might your goals best be achieved?

- What additional research might help to identify further strategies for improving the public health?

References are provided for all the reviews used and it is intended that the report will be regularly updated. The report contains about 350 densely typed, landscape pages. Copies can be obtained from the NHS Centre for Reviews and Dissemination. It can be accessed on the Web at www.york.ac.uk/inst/crd/wph.htm. 